Pacific Journal of Mathematic 


\title{
ON THE CONVERGENCE BEHAVIOUR OF TRIGONOMETRIC INTERPOLATING POLYNOMIALS
}

\author{
RICHARD P. GOSSELIN
}

1. Grünwald [1] and Marcinkiewicz [2] have shown by examples the existence of continuous functions for which the sequence of Lagrange interpolating polynomials taken at the Tchebysheff abcissas diverges at each point of $[-1,1]$. Marcinkiewicz constructed a function which actually proved an equivalent proposition, the existence of continuous functions for which the sequence of trigonometric interpolating polynomials taken at an even number of equidistant points centered at the origin diverges everywhere.

A similar result is known if for the $n$th polynomial the interpolating points are of the form $2 \pi i /(2 n+1), i=0, \pm 1, \cdots$; the sequence of interpolating polynomials corresponding to a certain continuous function, $f(x)$, diverges for every $x \neq 0(\bmod 2 \pi)$. The point $x=0$ must be excluded because it is of the form $2 \pi i /(2 n+1)$ for each $n$, and hence the $n$th polynomial must equal $f(x)$ there. (cf. Zygmund [3, p. 75]). We shall consider more generally the following sets of points

$$
\alpha+\frac{2 i}{2 n+1} \pi, \quad i=0, \pm 1, \pm 2, \cdots
$$

where $\alpha$ is any real number which is held fixed as $n$ varies. The points (1) are called the fundamental points of interpolation. We shall denote the $n$th trigonometric interpolating polynomial, that is, the uniquely defined polynomial of order not greater than $n$ which agrees with a given periodic function $f(x)$ at the points of $(1)$, by $I_{n}^{(\alpha)}(x ; f)$, except that we write $I_{n}(x ; f)$ for $I_{n}^{(0)}(x ; f)$.

In this paper, by refinements of the Marcinkiewicz example, along with adjustments for the new set of fundamental points, we show the strong dependence of the convergence behaviour of $I_{n}^{(\alpha)}(x ; f)$ for certain functions $f(x)$ on the number $\alpha$. For proper choice of $\alpha$, the convergence behaviour may be the worst possible, divergence for all $x \neq 0$ $(\bmod 2 \pi)$, whereas for the same function, another choice of $\alpha$ will lead to uniform convergence of the above sequence. We make these notions precise in the statements of our theorems.

THEOREM 1. For any real number $\alpha$, irrational with respect to $\pi$, there is a continuous function $f(x)$ for which the sequence $I_{n}(x ; f)$ diverges for all $x \neq 0(\bmod 2 \pi)$, but for which the sequence $I_{n}^{(\alpha)}(x ; f)$ converges uniformly.

Received May 6, 1954. 
2. The function in question, $f(x)$, is of the form

$$
\sum_{i=1}^{\infty} n_{i}^{-1 / 2} f_{n_{i}}(x)
$$

We shall define each $f_{n}(x)$ on certain points and impose some further general conditions which ensure that $I_{s}(x ; f)$ diverges for every $x \neq 0$ $(\bmod 2 \pi)$. This part of the construction is quite similar to that of the Marcinkiewicz example and is discussed in [3], so that our remarks here will be brief.

Each function $f_{n}(x)$ satisfies the following conditions: it is continuous and bounded by 1 ; smooth enough so that $I_{s}\left(x ; f_{n}\right)$ converges uniformly to it; but such that there is a bounded integral-valued function $\mu(x)$ defined on the closed intervals $[1 / n, \pi-1 / n],[\pi+1 / n$, $2 \pi-1 / n]$, and also for $x=\pi$ for which $\left|I_{\mu(x)}\left(x ; f_{n}\right)\right|>n$. Thus for each $n$, we choose $m$ so large that

$$
\int_{: \pi / m}^{1 / n} \frac{d \omega_{2 s+1}(\theta)}{2 \sin (\theta / 2)} \geq M(n), s \geq m ; \quad \begin{gathered}
\omega_{2 s+1}(\theta)=\theta_{i}^{(s)}, \theta_{i}^{(s)} \leq \theta<\theta_{i+1}^{(s)}, \\
\theta_{i}^{(s)}=\frac{2 \pi i}{2 s+1}, i=0, \pm 1, \pm 2, \cdots .
\end{gathered}
$$

$M(n)$ is some function of $n$ which we may take as large as we wish. Let $p_{1}, p_{2}, \cdots, p_{m}$ be integers all depending upon $n$ such that $m \leq p_{i}$ and

$$
m\left(2 p_{i}+3\right)^{3}<2 p_{i+1}+1, \quad i=1,2, \cdots, m-1 .
$$

For each $p$, let $S_{p}$ be the system of points $\theta_{i}^{(p)}, i=0,1,2, \cdots, 2 p$; and let $S_{p}(u)$ be the intersection of $S_{p}$ with the interval $[u, 2 \pi]$. We define $f_{n}(x)$ on $S_{p_{1}}$ as follows:

$$
f_{n}\left(\theta_{i}^{\left(p_{1}\right)}\right)= \begin{cases}(-1)^{i}, & \theta_{i}^{\left(p_{1}\right)} \in S_{p_{1}}(2 \pi / m) \\ 0, & \theta_{i}^{\left(p_{1}\right)} \in S_{p_{1}}-S_{p_{1}}(2 \pi / m) .\end{cases}
$$

Since $S_{p_{1}}$ and $S_{p_{1}+1}$ are disjoint, except for the point $x=0$, we may define $f_{n}(\theta)$ in the same way in $S_{p_{1}+1}$; that is equal to $(-1)^{i}$ if $\theta_{i}^{\left(p_{1}+1\right)}$ $\in S_{p_{1}+1}(2 \pi / m)$ and 0 elsewhere in $S_{p_{1}+1}$. Suppose now that $f_{n}(\theta)$ has been defined for $\theta \in S_{p i} \cup S_{p_{i}+1}, i=1,2, \cdots, k-1$. For the points of $S_{p_{k}} \cup S_{p_{k}+1}$ which coincide with points of $S_{p_{i}} \cup S_{p_{i}+1}, i=1,2, \cdots, k-1$, the original definition holds. For the remaining points of $S_{p_{k}} \backslash / S_{p_{k}+1}$, we define $f_{n}(\theta)$ as follows : 


$$
\begin{aligned}
f_{n}\left(\theta_{i}^{\left(p_{k}\right)}\right) & =\left\{\begin{array}{ll}
(-1)^{i}, & \theta_{i}^{\left(p_{k}\right)} \in S_{p_{k}}(2 \pi k / m) \\
0, & \theta_{i}^{\left(p_{k}\right)} \in S_{p_{k}}-S_{p_{k}}(2 \pi k / m)
\end{array} ;\right. \\
f_{n}\left(\theta_{i}^{\left(p_{k}+1\right)}\right) & = \begin{cases}(-1)^{i}, & \theta_{i}^{\left(p_{k}+1\right)} \in S_{p_{k}+1}(2 \pi k / m) \\
0, & \theta_{i}^{\left(p_{k}+1\right)} \in S_{p_{k}+1}-S_{p_{k}+1}(2 \pi k / m)\end{cases}
\end{aligned}
$$

Thus by recurrence, $f_{n}(\theta)$ is defined for all points of $S_{p_{i}} \cup S_{p_{i}+1}, i=1$, $2, \cdots, m-1 . f_{n}(\theta)$ may be defined arbitrarily elsewhere except that it must be continuous, bounded by 1 , and smooth enough to ensure the uniform convergence of $I_{s}\left(x ; f_{n}\right)$.

Every point $x$ of the interval $[1 / n, 2 \pi-1 / n]$ belongs to some interval $[2(k-1) \pi / m, 2 k \pi / m], k=1,2, \cdots, m-1$. For $x$ in the $k$ th interval, we may write according to a well known formula

$$
\begin{aligned}
& I_{s}\left(x ; f_{n}\right)=\frac{1}{\pi} \int_{0}^{2 \pi} f_{n}(\theta) D_{s}(x-\theta) d \omega_{2 s+1}(\theta)
\end{aligned}
$$

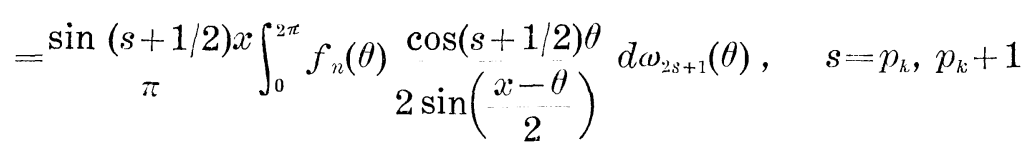

where

$$
D_{s}(x)=\frac{\sin (s+1 / 2) x}{2 \sin (x / 2)} .
$$

By arguments similar to those in [2], we may show, using (2), that

$$
\left|I_{s}\left(x ; f_{n}\right)\right| \geq\left|\begin{array}{c}
\sin (s+1 / 2) x \\
\pi
\end{array}\right| M(n)+O(n), \quad s=p_{k}, p_{k}+1 .
$$

If $x=\pi$, then $\sin (s+1 / 2) x= \pm 1$. If $x$ belongs to one of the intervals $[1 / n, \pi-1 / n, \pi+1 / n, 2 \pi-1 / n]$, then either $\left|\sin \left(p_{k}+1 / 2\right) x\right| \geq 1 / \pi n$ or $\left|\sin \left(p_{k}+3 / 2\right) x\right| \geq 1 / \pi n$. This shows with suitable choice of $M(n)$ that

$$
\left|I_{s}\left(x, f_{n}\right)\right|>n, \quad x \in[1 / n, \pi-1 / n],[\pi+1 / n, 2 \pi-1 / n], \text { or } x=\pi
$$

where $s$ is chosen to be $p_{k}$ or $p_{k}+1$ for some $k$. The $n_{i}$ 's are spread out so sparsely that the following conditions are satisfied:

$$
\sum_{i=1}^{\infty} n_{i}^{-1 / 2}<\infty ; \quad p_{m-1}\left(n_{i}\right) \sum_{k=i+1}^{\infty} n_{k}^{-1 / 2}<1 ; \quad \text { and } \quad\left|I_{s}\left(x ; f_{n_{k}}\right)\right|<2, m\left(n_{i}\right) \leq s, k<i .
$$

Because of the first condition, $f(x)$ is continuous, and the last can be satisfied by the uniform convergence of $I_{s}\left(x ; f_{n}\right)$. By well known arguments (cf., for example, Zygmund [3, pp. 79, 80]), these conditions are sufficient to make $I_{s}(x ; f)$ diverge for every $x \neq 0(\bmod 2 \pi)$. 
3. Our own proof depends upon defining more explicitly each $f_{n}(x)$ throughout the interval $[0,2 \pi]$. Let $T(n)$ denote the set of points where $f_{n}(x)$ has already been defined to be \pm 1 . Let $r=r_{n}$ be the number of points of $T(n)$. For each $s$, there exists an integer $\nu(s)$ such that all the points

$$
x_{j}(s)=\alpha+\frac{2 i \pi}{2 s+1}+\frac{2 \nu(s) \pi}{2 s+1}, \quad j=0,1,2, \cdots, 2 s
$$

belong to the interval $[0,2 \pi]$. All of the numbers

$$
\begin{array}{ll}
x_{j}(s)-\frac{2 i \pi}{2 p+1}, \quad j=0,1, \cdots, 2 s ; s=1,2, \cdots, s_{1} \\
\quad i=0,1, \cdots, 2 p ; p=p_{1}, p_{2}, \cdots, p_{m-1}, p_{1}+1, \cdots, p_{m-1}+1
\end{array}
$$

are different from 0 , else our hypothesis about $\alpha$ would be violated. Let $\gamma\left(s_{1}\right)$, depending upon $n$, be the minimum of the absolute value of these numbers. Choose disjoint intervals $I_{\xi}$ of length $2 \eta=2 \eta_{n}$ centered about each point $\xi$ of $T(n)$. We choose $\eta$ so small that $I_{\xi}$ contains no other points of $S_{p_{i}} \cup S_{p_{i}+1}, i=1,2, \cdots, m-1$. Let $s_{1}$ be so large that (a) $2 r / r(2 s+1)<1$ for $s \geq s_{1}$. Now in each interval $I_{\xi}$, we let $f_{n}(x)$ equal a "roof" function, $\pm \lambda_{\delta}(x-\xi)$ where $\lambda_{\delta}(0)=1 ; \lambda_{\delta}(x)=0$ if $|x| \geq \delta$, and $\lambda_{\delta}(x-\xi)$ is linear from $\xi-\delta$ to $\xi$ and from $\xi$ to $\xi+\delta$. The plus or minus sign is of course chosen in accordance with the original definition of $f_{n}(x)$ at $\xi$. Let $\delta=\delta_{n}$ be so small that (b) $r \delta<\eta$ and (c) $\delta<\gamma\left(s_{1}\right)$. Elsewhere, we define $f_{n}(x)$ to be 0 . Condition (c) guarantees that $f_{n}(x)$ will be 0 at all points $x_{j}(s), j=0,1, \ldots, 2 s ; s \leq s_{1}$. Since $f_{n}(x)$ satisfies a Lipschitz condition, both $I_{s}\left(x ; f_{n}\right)$ and $I_{s}^{(\alpha)}\left(x ; f_{n}\right)$ converge uniformly to $f_{n}(x)$.

4. We now proceed to show that $\left|I_{s}^{(\alpha)}\left(x ; f_{n}\right)\right|<A$ for all $x$ in $[0,2 \pi]$ where $A$ is a constant independent of $x, s$, and $n$. We have

$$
\begin{aligned}
I_{s}^{(\alpha)}\left(x ; f_{n}\right) & =\frac{1}{\pi} \int_{x-\pi}^{x+\pi} f_{n}(\theta) D_{s}(x-\theta) d \omega_{2 s+1}(\theta) \\
& =\frac{2}{2 s+1} \sum_{j} f_{n}\left(x_{j}(s)\right) D_{s}\left(x-x_{j}(s)\right) .
\end{aligned}
$$

If $s \leq s_{1}$, then $I_{s}^{(\alpha)}\left(x ; f_{n}\right)$ is 0 by condition (c) on $\delta$. If $s>s_{1}$, we write $I_{s}^{(\alpha)}\left(x ; f_{n}\right)=I_{s, 1}^{(\alpha)}\left(x ; f_{n}\right)+I_{s, 2}^{(\alpha)}\left(x ; f_{n}\right)=\frac{2}{2 s+1}\left\{\sum_{1}+\sum_{2}\right\} f_{n}\left(x_{j}(s)\right) D_{s}\left(x-x_{j}(s)\right)$

where $I_{s, 2}^{(\alpha)}\left(x ; f_{n}\right)$ consists of those terms of the sum corresponding to the points $x_{j}(s)$ which belong to the interval $I_{\xi}$ containing $x$ for some 
$\xi$ (if $x$ belongs to one such interval), and $I_{s, 1}^{(\alpha)}\left(x ; f_{n}\right)$ consists of the remaining terms of the sum. We have

$$
\left|I_{s, 1}^{(\alpha)}\left(x ; f_{n}\right)\right| \leq \frac{\pi}{2 s+1} \sum_{1} \frac{1}{\left|x-x_{j}(s)\right|} .
$$

Let $\eta-\delta=\beta=\beta_{n}$. Then $\left|x-x_{j}(s)\right| \geq \beta$ for the terms of $\sum_{1}$, whether $x$ belongs to any interval $I_{\xi}$ or not. Now let $k_{n}(s)$ be the number of terms of $\Sigma_{1}$. It follows that

$$
\left|I_{s, 1}^{(\alpha)}\left(x ; f_{n}\right)\right| \leq \frac{\pi k_{n}(s)}{(2 s+1) \beta} .
$$

To estimate $k_{n}(s)$, consider all of the intervals $I_{\xi}$, except the one which contains $x$ (if there is such). In each $f_{n}(x)$ is different from 0 only on a subinterval of length $2 \delta$. Since successive fundamental points are separated by a distance $2 \pi /(2 s+1)$, there are at most $\langle\delta(2 s+1) / \pi\rangle+1$ distinct points $x_{j}(s)$ of the sum $\sum_{1}$ in this interval, where $\langle y\rangle$ denotes the least integer greater than or equal to $y$. Since there are not more than $r$ such intervals, we have that

$$
k_{n}(s) \leq \frac{\delta r(2 s+1)}{\pi}+2 r
$$

and from (3)

$$
\left|I_{s, 1}^{(\alpha)}\left(x ; f_{n}\right)\right| \leq \frac{\delta r}{\beta}+\frac{2 \pi r}{\beta(2 s+1)}
$$

Condition (b) implies that $\delta<\eta / 2$ for $r \geq 2$, and hence that $\beta>\eta / 2$. Thus $\delta r / \beta<2 \delta r / \eta<2$, the latter inequality also following from condition (b). For the second quantity on the right side of (4), we have $r /(2 s+1) \beta<2 r /(2 s+1) \eta<1$, the latter inequality being condition (a), which holds since $s>s_{1}$. Combining these results, we obtain from (4) that

$$
\left|I_{s, 1}^{(\alpha)}\left(x ; f_{n}\right)\right| \leq 2+\pi .
$$

If $x$ belongs to none of the intervals $I_{\xi}$, then the estimate (5) of $I_{s, 1}^{(\alpha)}\left(x ; f_{n}\right)$ will serve also for $I_{s}^{(\alpha)}\left(x ; f_{n}\right)$. If $x$ does belong to one of the intervals $I_{\xi}$, then

$$
\begin{aligned}
I_{s, 2}^{(\alpha)}\left(x ; f_{n}\right)= & \pm \frac{1}{2 s+1} \sin [(s+1 / 2)(x-\alpha)] \\
& \cdot \sum_{2} \lambda_{\delta}\left(x_{j}(s)-\xi\right) \frac{(-1)^{j}}{2 \sin \left[\left(x-x_{j}(s)\right) / 2\right]}
\end{aligned}
$$


where the index $j$ corresponds to the points $x_{j}(s)$ of the interval $I_{\xi}$. The sum in (6) can be written as $\sum_{2,1}+\sum_{2,2}+\sum_{2,3}$ where $\sum_{2,1}$ consists of those terms of $\sum_{2}$ for which $\left|x-x_{j}(s)\right| \leq 2 \pi \mid(2 s+1), \quad \sum_{2,2}$ of the remaining terms for which $\sin \left[\left(x-x_{j}(s)\right) / 2\right]<0$, and $\Sigma_{2,3}$ of the remaining terms for which $\sin \left[\left(x-x_{j}(s)\right) / 2\right]>0$. Since there are at most three terms of $\sum_{2,1}$,

$$
\left|\begin{array}{c}
1 \\
2 s+1
\end{array} \sin [(s+1 / 2)(x-\alpha)] \sum_{2,1} \lambda_{s}\left(x_{j}(s)-\xi\right) \frac{(-1)^{j}}{2 \sin \left[\left(x-x_{j}(s)\right) / 2\right]}\right| \leq 3 .
$$

The sum of successive terms of $\sum_{2,2}$ is

$$
\frac{\lambda_{\delta}\left(x_{j+1}(s)-\xi\right)}{2 \sin \left[\left(x-x_{j+1}(s)\right) / 2\right]}-\frac{\lambda_{\delta}\left(x_{j}(s)-\xi\right)}{2 \sin \left[\left(x-x_{j}(s)\right) / 2\right]} .
$$

All of the terms, except possibly for two, of $\sum_{2,2}$ can be paired as in (8) so that $\lambda_{\delta}(x-\xi)$ is linear for $x_{j}(s) \leq x \leq x_{j+1}(s)$. For these two terms $4 s+2$ is a bound. For the remaining, we apply the mean value theorem to obtain that the absolute value of the difference (8) is not greater than

$$
\frac{\pi^{2}}{\delta(2 s+1)\left|x-x_{j}(s)\right|}+\frac{\pi^{3}}{2(2 s+1)\left|x-x_{j}(s)\right|^{2}}
$$

and so

$$
\begin{aligned}
& \begin{array}{c}
1 \\
2 s+1
\end{array}\left|\sum_{2,2} \lambda_{\delta}\left(x_{j}(s)-\xi\right) \frac{(-1)^{j}}{2 \sin \left[\left(x-x_{j}(s)\right) / 2\right]}\right| \\
& \quad \leq 2+\frac{\pi^{2}}{\delta(2 s+1)^{2}} \sum_{2,2} \frac{1}{\left|x-x_{j}(s)\right|}+\frac{\pi^{3}}{2(2 s+1)^{2}} \sum_{2,2} \frac{1}{\left|x-x_{j}(s)\right|^{2}} .
\end{aligned}
$$

Since the number of terms of $\sum_{2,2}$ is not greater than $2+\delta(2 s+1) / \pi$ and the smallest possible value for $\left|x-x_{j}(s)\right|$ is $2 \pi /(2 s+1)$, the second term on the right side of (9) is not greater than $1 / 2+\pi / \delta(2 s+1)$. If $(2 s+1) \delta / \pi<1$, then there is at most one term in the sum of (6) so that one would serve as a bound for $\left|I_{s, 2}^{(\alpha)}\left(x ; f_{n}\right)\right|$. Hence, we assume otherwise, and $\pi / \delta(2 s+1) \leq 1$. For the third term on the right side of (9), since the smallest possible value for $\left|x-x_{j}(s)\right|$ is $2 \pi /(2 s+1)$, and since successive terms differ by $2 \pi /(2 s+1)$, we have

$$
\frac{\pi^{3}}{2(2 s+1)} \sum_{2,2} \frac{1}{\left|x-x_{j}(s)\right|^{2}} \leq \frac{\pi^{3}}{2(2 s+1)^{2}}\left[\frac{(2 s+1)^{2}}{(2 \pi)^{2}}+\frac{(2 s+1)^{2}}{(4 \pi)^{2}}+\cdots\right]=c,
$$

a constant. Hence, collecting these results, we see, using (9), that

$$
\frac{1}{2 s+1}\left|\sum_{2,2} \lambda_{\delta}\left(x_{j}(s)-\xi\right) \frac{(-1)^{j}}{2 \sin \left[\left(x-x_{j}(s)\right) / 2\right]}\right| \leq c+7 / 2 .
$$


A similar result holds for $\sum_{2,3}$, so that from (6), (7), and (10), we see that $\left|I_{s, 2}^{(\alpha)}\left(x ; f_{n}\right)\right|$ is bounded by a constant $A_{2}$ independent of $x, s$, and $n$. From $(5),\left|I_{s, 1}^{(\alpha)}\left(x ; f_{n}\right)\right|$ is bounded by a constant $A_{1}$, independent of $x, s$, and $n$. Thus, $\left|I_{s}^{(\alpha)}\left(x ; f_{n}\right)\right|$ is bounded by a constant $A=A_{1}+A_{2}$.

5. The last result shown together with the uniform convergence of $I_{s}^{(\alpha)}\left(x ; f_{n}\right)$ gives

$$
\begin{aligned}
\left|I_{s}^{(\alpha)}(x ; f)-f(x)\right| & \leq \sum_{i=0}^{N} n_{i}^{-1 / 2}\left|I_{s}^{(\alpha)}\left(x ; f_{n_{i}}\right)-f_{n_{i}}(x)\right|+\sum_{i=N+1}^{\infty} n_{i}^{-1 / 2}\left|I_{s}^{(\alpha)}\left(x ; f_{n_{i}}\right)-f_{n_{i}}(x)\right| \\
& \leq \varepsilon \sum_{i=1}^{N} n_{i}^{-1 / 2}+(A+1) \sum_{i=N+1}^{\infty} n_{i}^{-1 / 2}
\end{aligned}
$$

for $s$ large enough. Since the right hand side is arbitrarily small with $\varepsilon$ and $1 / N$, our theorem is proved.

6. With a slight modification of the previous argument, we may establish the following theorem.

THEOREM 2. There is a continuous function $f(x)$ such that $I_{s}(x ; f)$ diverges for every $x \neq 0(\bmod 2 \pi)$ while for almost every number $\alpha$, $I_{s}^{(\alpha)}(x ; f)$ converges uniformly.

Our function, $f(x)$, will be of the same form as in Theorem 1, $\sum_{i=1}^{\infty} n_{i}^{-1 / 2} f_{n_{i}}(x)$, where the $f_{n_{i}}(x)$ are sums of nonoverlapping roof functions. Let $I_{\xi}, \eta, s_{1}$ be defined as before. Consider

$$
\text { (11) }-\begin{gathered}
2 \pi l \\
2 s+1
\end{gathered}+\theta_{i}^{(n)}, \begin{aligned}
& l=-2 s-1, \cdots, 0,1, \cdots, 2 s ; s \leq s_{1} \\
& i=0,1, \cdots, 2 p ; p=p_{1}, p_{1}+1, \cdots, p_{m}, p_{m}+1
\end{aligned}, \theta_{i}^{(p)}=\frac{2 \pi i}{2 p+1} .
$$

Suppose that there are $\tau=\tau_{n}$ such numbers. Choose symmetric neighbourhoods of length $2 / \tau^{2}$ about each, and denote the set which consists of the sum of these neighbourhoods by $R_{n}^{\prime}$. Let $\alpha$ belong to $R_{n}$ (complement of $\left.R_{n}^{\prime}\right), 0 \leq \alpha \leq 2 \pi$. Let $\nu(s)$ denote the least integer such that $\alpha+2 \pi \nu(s) /(2 s+1) \geq 0$. Clearly $-2 s-1 \leq \nu(s) \leq 0$. Then the numbers

$$
\alpha+\left(\frac{2 \pi j}{2 s+1}\right)+\left(\begin{array}{c}
2 \pi \nu(s) \\
2 s+1
\end{array}\right), \quad j=0,1, \cdots, 2 s
$$

belong to the interval $(0,2 \pi)$ and so are our $x_{j}^{(\alpha)}(s)$. Also the numbers $j+\nu(s)$ are included in the numbers $l$ of (11) for $s \leq s_{1}$ since $-2 s-1 \leq$ $j+\nu(s) \leq 2 s$. We choose $\delta$ such that it satisfies $r \delta<\eta$ (Condition (b) of Theorem 1) and in addition $\left(\mathrm{c}^{\prime}\right) \delta<1 / \tau^{2}$. We have 


$$
x_{j}^{(\alpha)}(s)-\theta_{i}^{(p)}=\alpha-\left[-\frac{2 \pi l}{2 s+1}+\theta_{i}^{(p)}\right]
$$

for some $l$ such that $-2 s-1 \leq l \leq 2 s, s \leq s_{1}$. Hence $\left|x_{j}^{(\alpha)}(s)-\theta_{i}^{(p)}\right| \geq 1 / \tau^{2}>\delta$ by $\left(\mathrm{c}^{\prime}\right)$ so that $I_{s}^{(\alpha)}\left(x ; f_{n}\right)=0$ for all $x, s \leq s_{1}$, and all $\alpha$ in $R_{n}$. To show that $I_{s}^{(\alpha)}\left(x ; f_{n}\right)$ is bounded for all $s$ and all $\alpha$ in $R_{n}$, we employ the previous argument, which, beyond this point, used nothing about $\alpha$.

Considering only the portions of $R_{n}$ and $R_{n}^{\prime}$ in $(0,2 \pi)$, we have

$$
\left|R_{n}^{\prime}\right| \leq \frac{2}{\tau_{n}} ; \sum_{i=1}^{\infty}\left|R_{n_{i}}^{\prime}\right| \leq 2 \sum_{i=1}^{\infty} \frac{1}{\tau_{n_{i}}}<\infty
$$

for the $n_{i}$ spread out sufficiently. Hence, except for a set of measure 0 , every $\alpha$ belongs to at most a finite number of sets $R_{n_{i}}^{\prime}$ and so to every $R_{n_{i}}$ for $i$ large enough.

The author would like to acknowledge his indebtedness to Professor A. Zygmund for suggesting to him a result of the type of Theorem 1. Theorem 2 was established in response to a question raised by Professor E. G. Straus.

\section{REFERENCES}

1. G. Grünwald, Uber Divergenzerscheinungen der Lagrangeschen Interpolationspolynome stetiger Funktionen, Ann. of Math., 37 (1936), 908-918.

2. J. Marcinkiewicz, Sur la divergence des polynomes d'interpolation, Acta Univ. Szeged, 8 (1936-37), 131-135.

3. A. Zygmund, Trigonometrical Interpolation, (mimeographed notes). University of Chicago, 1950.

UNIVERSITY OF CONNECTICUT

STORRS, CONNECTICUT 
.././. ./FrontMatter/paper .pdf 


\section{Pacific Journal of Mathematics}

Nesmith Cornett Ankeny and Theodore Joseph Rivlin, On a theorem of S.

Bernstei........................................ 849

Louis Auslander, The use of forms in variational calculation .......... 853

Paul Civin, Abstract Riemann sum . .......................... 861

Paul Civin, Some ergodic theorems involving two operator ............ 869

Eckford Cohen, The number of solutions of certain cubic congruence .... . 877

Richard M. Cohn, Specializations over difference field .............. 887

Jean Dieudonné, Pseudo-discriminant and Dickson invarian . . ......... 907

Ky Fan, A comparison theorem for eigenvalues of normal matrice ........ 911

Richard P. Gosselin, On the convergence behaviour of trigonometric interpolating polynomial ........................... 915

Peter K. Henrici, On generating functions of the Jacobi polynomial . . . . . . . 923

Meyer Jerison, An algebra associated with a compact grou ............ 933

Wilhelm Magnus, Infinite determinants associated with Hill's equatio . . . . . 941

G. Power and D. L. Scott-Hutton, The slow steady motion of liquid past a semi-elliptical bos................................. 953

Lyle E. Pursell, An algebraic characterization of fixed ideals in certain function ring .................................... 963

C. T. Rajagopal, Additional note on some Tauberian theorems of O. Szás . . 971 Louis Baker Rall, Error bounds for iterative solutions of Fredholm integral

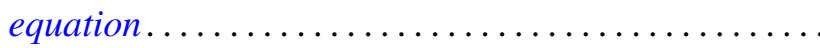

Shigeo Sasaki and Kentaro Yano, Pseudo-analytic vectors on

pseudo-Kählerian manifold ......................

Eugene Schenkman, On the tower theorem for finite group

P. Stein and John E. L. Peck, On the numerical solution of Poisson's equation over a rectangl ........................

Morgan Ward, The mappings of the positive integers into themselves which preserve divisio .

Seth Warner, Weak locally multiplicatively-convex algebra 1025

Louis Weisner, Group-theoretic origin of certain generating function .... 